\title{
Estimation of Age from Face Images
}

\author{
Suvarna E. Padme ${ }^{1}$, P. S. Desai ${ }^{2}$ \\ Department of Computer Engineering, SKNCOE, Pune, Savitribai Phule Pune University, Pune.
}

\begin{abstract}
An important personal trait is human age, can be directly inferred by the different patterns appear on the face. The recently use topics are the computer-based age synthesis and estimation from faces and has the various applications such as in customer relationship management, biometrics, security control, cosmetology. Face recognition is challenging and interesting area in real time applications. An age estimation is the estimation of age from the face image with the exact age in year or the age range. In this paper we survey the complete age synthesis from face images techniques. Existing models, various algorithms, face images databases, protocols, techniques, future discussion are discussed in this paper.
\end{abstract}

Keywords: Age progression, age estimation, face aging, age synthesis

\section{Introduction}

The human face has the attributes such as gender, age, personal identity, facial expressions. The face image processing techniques has the various applications in computer vision fields and graphics. There are two fundamental problems in the above face image processing techniques [1],[2]:

- Face image synthesis- The image of face with single or mixed facial attributes such as identity of person, face expression, gender and age.

- Face image analysis- The image of face with facial attributes such as identity of person, face expression, gender and age.

The methods used for face recognition is Gabor wavelet tool like ANN for face recognition. Face recognition is an important part of human perception system and is task for humans. Face recognition is one of the application of the pattern recognition and image analysis.

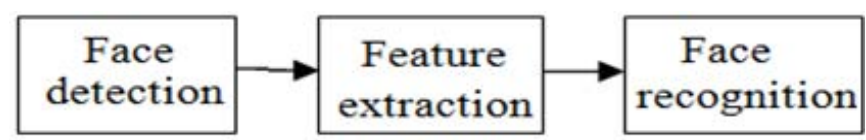

Figure 1: Face recognition system

The above figure 1.1 shows the face recognition system, in face detection the face is extracted from the scene and sees whether the face is present in that scene or not. In feature extraction step the face regions, variations and angles are extracted from face image. In face recognition step the comparison method and classification algorithms are used for the recognition of human face.

\section{Motivation}

The real world applications are rich and attractive. Different peoples have different aging process which is determined not only by person's genes but also from the other factors like living style, sociality, nature. Males and females have different aging patterns because of using wakeups and different accessories. Many female faces show younger appearance by using makeup. This paper proposes the whole survey of the various operations and the state-of-the-art techniques.

\section{Literature Survey}

The studies are mostly aimed at age synthesis, i.e. changing the appearance of a rendered face to show proper effects of aging. Some of these works are useful in the determination of appropriate facial features for age estimation. We are present the brief information of some of paper.

A survey paper on topic age synthesis and estimation via faces by Y. Fu, G. Guo, and T. S. Huang [3], proposed age synthesis and estimation from faces. In this survey paper, these techniques are used such as face aging, age estimation, age synthesis, age progression.

Another paper on topic age estimation from face images: Human vs. machine performance by H. Han, C. Otto, and A. $\mathrm{K}$. Jain proposed a hierarchical approach for automatic age estimation, and provide an analysis of how aging influences individual facial components. Experimental results on the FG-NET, MORPH Album2, and PCSO databases show that eyes and nose are more informative than the other facial components in automatic age estimation. The methods used in this paper are face preprocessing, facial Component Localization and feature extraction.

\section{Human Aging from Faces}

The problem with aging isn't that it's completely horrible. Face aging is generally slow and irreversible process. There are some general changes when people are aging differently and aging show different aging patterns. From child birth to adulthood the changes occur in the face size. The face size changes from the child to adulthood image. The face size changes that is the shape of eyes, nose, mouth, eyebrows and lips changes from child to adulthood.

\section{Volume 4 Issue 12, December 2015}




\section{International Journal of Science and Research (IJSR) \\ ISSN (Online): 2319-7064}

Index Copernicus Value (2013): 6.14 | Impact Factor (2014): 5.611

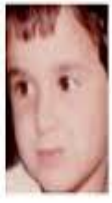

3 years old (single input)

Figure 2: Face aging cases from 3 -100 years

The craniofacial growth is important in face aging due to this the cheeks are extend to larger areas and the chin becomes more protrusive. The facial skin does not change too much as compared with the craniofacial growth. The hairs present on the face are becomes thick and bushy. The change in the skin color occur in little bit amount. During aging from adulthood to old age, the changes occur in the skin aging. The figure 2 shows the face aging cases from 3-100 years. face aging during old age period is the sagging of the skin such as dropping cheek and lower eyelid bags. Also the aging during this period cause loss of control of muscles which result in facial movements and behaviors. Face aging is related with the attractiveness and distinctiveness. The young faces are more attractive and less distinctive than old faces. When the degree of caricature increases then the distinctiveness increases and the attractiveness decreases. O'Toole et al. [4], applied a facial caricature algorithm on 3D face images and conclude that the average face is more attractive and younger than individual face.

\section{Age Estimation Methods}

The component-based representation is used for the estimation of the age from face images. The actual estimation of the age from face image is as shown in the figure 3.
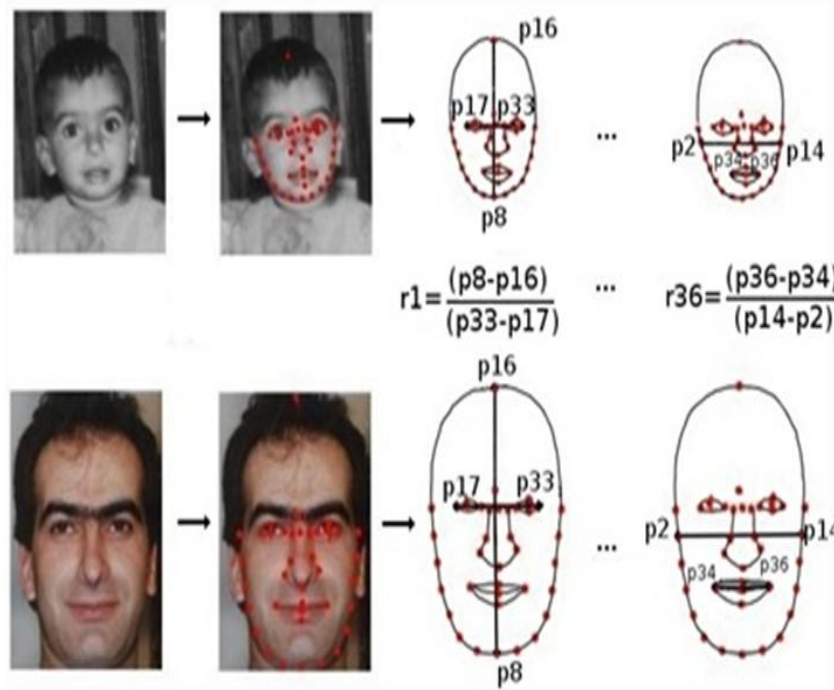

$r 36=\frac{(p 36 \cdot p 34)}{(p 14 \cdot p 2)}$

Figure 3: Estimation of age from face images

As shown in the figure 3 the main four steps are present: preprocessing, facial component localization, feature extraction, and hierarchical age estimation.

\subsection{Preprocessing of Face}

The face images are present in the face aging databases, e.g. FGNET in this databases the stored images are captured using different techniques such as scanned photographs or digital cameras. The images present in the FGNET databases are grey scale or color. When we apply preprocessing on the face image then first we convert that face mage into grey scale image. There are two rotations are present i.e. in -scale and out-of -scale. To improve the accuracy of the facial components, a non reflective similarity transformation is applied to normalize each face image based on two eyes. The FGNET databases have 68 landmarks including the eye centers for each face image. While in the MORPH database do not provide the landmarks, we automatically detect the eye centers by using Face VACS SDK.

\subsection{Localization of Facial Components}

We propose a method which is based on ASM to localize individual components of the face to automatically detect a landmarks on face followed by component localization corresponding to the forehead, eyes, nose and mouth. Figure 4 shows the original, preprocessed and manual facial landmarks.

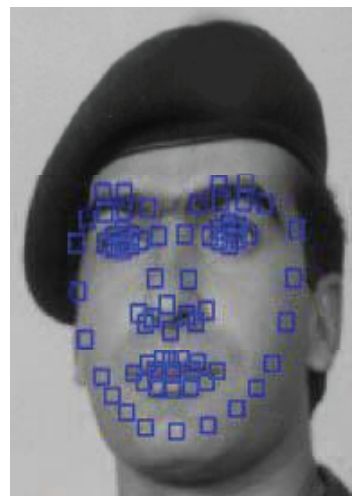

Figure 4: Facial component localization

\subsection{Feature Extraction}

Serre et al. [5], proposed a biologically inspired model (BIM) for object recognition which has also found in automatic age estimation. Instead of extracting biologically inspired features (BIF) from a holistic face like we extract BIF features from individual facial components. The BIM contains the two layers of computational unit, where simple $\mathrm{S} 1$ units are followed by complex $\mathrm{C} 1$ units. The $S 1$ units correspond to the classical simple cells of Hubel and Wiesel found in the primary visual cortex (V1).

\subsection{Hierarchical age estimation:}

The hierarchical age estimation method is as shown in figure 3. Each facial component is first classified into four disjoint age groups using the binary decision tree based on SVM. SVM age repressors is used to predict the final age. 


\section{International Journal of Science and Research (IJSR) \\ ISSN (Online): 2319-7064}

Index Copernicus Value (2013): 6.14 | Impact Factor (2014): 5.611

\subsection{Age Estimate Fusion}

Age estimation performance can be improved by fusing different features for different age groups.

\section{Age Estimation from Faces}

As compared with the ongoing research on age synthesis, there are specifically various publications on the age estimation. This above situation is caused by three reasons: 1) Age estimation is derived from different application scenarios, it can either as multiclass classification problem or a regression problem. 2) It is hard to collect the chronometrical images of an individual for the large aging databases. 3) The age progression on faces is uncontrollable and personalized. The above characteristics of aging variation cannot be captured accurately due to the prolific and varying information by human faces. The existing age estimation systems consists of basically two modules: age image representation and age estimation techniques.

\subsection{Age image representation:}

The age image representation consists of the five different models which is given below.

\subsubsection{Anthropometric Models:}

The Kwon and Lobo [6], published the paper in the area of age classification from facial images. The main aspect of this approach is to consult studies in cranio-facial development studies. The theory of cranio-facial consists of the mathematical model to describe the growth of person's head from childhood to adulthood. Farkes [6], defined the face anthropometry in terms of the 57 landmarks and the fiducial points on the face for different ages from childhood to adulthood. There are two reasons that the people do not use this mathematical model for age estimation:1) The mathematical model cannot characteristize the head profile in natural way, 2) From 2D face images the head profile is difficult to measure. The Kwon and Lobo computed six ratios of distances on frontal face images to distinguish baby and adult person.

The age estimation approaches based on the anthropometry can only deal with the young faces, the human shape of the head do not change much more in adulthood. The Kwon and Lobo computed wrinkles from the face images to distinguish baby from the adulthood. They compute the wrinkles in different regions i.e. forehead, cheek, nose. The anthropometry model is used in both the age group.

\subsubsection{Active Appearance Model}

The active appearance model is used for coding face images. Lanitis et.al.[7], also tried to use different classifiers for age estimation. In comparison with the anthropometric model the AAMs is used in general images rather than young ages. The AAMs based approach considers shape and texture of the images rather than considering the facial geometry of the face as consider in the anthropometric model.

\subsubsection{Aging Pattern Subspace}

To model the aging process instead of dealing with each aging face separately, we can deal with sequence of aging faces. This idea is called as Aging pattern subspace (AGES).An aging pattern is defined as the sequence of face images coming from the same person. The aging pattern of the face images of all ages are available for all individuals is called as complete age patterns, otherwise it is called as incomplete age pattern. This aging method is works in two stages: the learning stage and the age estimation stage. In the first learning stage, PCA technique is used to obtain subspace representation.

In the aging pattern subspace, each face is first encoded by using the AAMs method. The experiment for the AGES method was performed on the FG-NET aging database. In AGES method, the problem is that in order to estimate the age of an input face image, the AGES method assumes that there exist the image of the same person with different age or at least similar aging pattern is present in the database.

\subsubsection{Appearance Models}

The facial feature extraction is focused by appearance model. Two types of features is used in age estimation systems first is global feature and second is local feature. Hayashi et al. consider both shape and texture feature to characterize each face image. To characterize facial features a semantic level of description was also used. Gender estimation was also implemented to improve the age estimation. The aging patterns are different for male and female. Fukai and Takimoto [8], presented to extract feature spectrum from facial appearance by using FFT and Genetic algorithm is used for feature selection.

\section{Age Estimation Algorithms}

The age estimation can be considered as a classification problem. The age numbers are a set of sequential values e.g. $0,1,2, \ldots$ thus age estimation can also be the regression problem. It is more effective to consider the age estimation is classification or regression problem?

\subsection{Classification:}

Lanitis et al. [7], evaluated the performance for different classifiers for age estimation, including Artificial Neural Network (ANN), nearest neighbor classifier. The AAM method is used for the representation for the face images. The quadratic function is actually a regression function. This function is used to relate the face representations to age labels and is also called as quadratic function classifier [7]. The author suggested that first do the clustering, and then to refine the age estimation in a hierarchical fashion. The authors also compared age estimation with the computer to the human. The classification of the age group is determined by fitting the test image to the Gaussian model. In the age group classification of 5-year, their system achieves the accuracies of 50 percent for male and 43 percent for female. In the age group classification of 10-year, their system achieves the accuracies of 72 percent of male and 63 percent for female. In the age group classification of 15-year, their 


\section{International Journal of Science and Research (IJSR) \\ ISSN (Online): 2319-7064}

Index Copernicus Value (2013): 6.14 | Impact Factor (2014): 5.611

system achieves the accuracies of 82 percent for male and 74 percent for female.

\subsection{Regression}

By considering age estimation is a regression problem, Lanitis [7] investigated three formulations for the aging function i.e. linear, quadratic and cubic. Inspired from the Lantis's work, Fu et al. [9], used a multiple linear regression function to fit the CEA aging manifold, this function achieves the improvements over some existing techniques. The Support Vector Regression (SVR) was applied on the OLPP age manifold for age estimation. Yan et al.[10],[11], used a Semi Definite Programming (SDP) formulation to solve the regression problem for age estimation. The authors showed that the SDP formulation for age regression gives better results than the quadratic regression function. The SDP is expensive and an effective tool. The solution to SDP may be difficult to achieve, when the size of the training set is large. The Expectation-Maximization (EM) algorithm was used to solve the regression problem. This algorithm is also used to speed up the optimization process.

\section{Applications}

There are many applications for age synthesis.

\subsection{Customer Relationship Management (CRM)}

The CRM [12] is use for the information technology and the interaction with the customers and communicate with that customer individually. Different groups of customer have very different consuming habits. The personal information can be obtained from all customer groups, and maintain the long term relation with the customer. For example, a fast food shop owner want to know what percentage of the age groups are interested in particular product (e.g. sandwiches), the mobile company want to know that what percentage of the age group is interested in particular product. however, with the help of computer based automatic age estimation system the camera can capture the photo of the customer and automatically detect the age of that person and labeling age groups.

\subsection{Security Control}

Security control issues are more decisive in our day-to-day life. With the help of the camera which is used for the age estimation ,stop the underage (less than 18 years) people to enter in the bar, stop the underage people to buy the tobacco and also with the help of the camera we cannot provide driving license to the underage people.

\subsection{Biometrics}

Age estimation is the type of biometrics, that provide the user's identity information. The primary biometric features are the face, iris scan, voice scan, retina scan and thumb impression which is used in the biometrics.

\subsection{Entertainment}

Aging is the popular effects in making film. With the help of these aging effects the actor's appearance can be transformed from young to old and from old to young.

\subsection{Cosmetology}

Many people want to have their faces look younger. The cosmetic surgery and skin care is the popular beauty treatment in cosmetology. The beauticians and surgeons use the computer aided system in the cosmetology.

\section{Conclusion}

This paper presented a complete survey of the techniques for age synthesis and estimation of age from face images. The different age synthesis and estimation algorithms can be effectively applied to various applications. In this paper we presented proposed age estimation method which is used to find the age from the facial images.

\section{Acknowledgement}

I wish to thank Prof. P.S.Desai for giving me proper guidance and suggestions regarding this work.

\section{References}

[1] L.A. Zebrowitz, Reading Faces: Window to the Soul? Westview Press, 1997.

[2] A. Gallagher and T. Chen, "Estimating Age, Gender, and Identity Using First Name Priors," Proc. IEEE Conf. Computer Vision and Pattern Recognition, 2008.

[3] G. Guo, Y. Fu, C. Dyer, and T.S. Huang, "Image-Based Human Age Estimation by Manifold Learning and Locally Adjusted Robust Regression," IEEE Trans. Image Processing, vol. 17, no. 7, pp. 1178-1188, July 2008.

[4] A.J. O'Toole, T. Price, T. Vetter, J.C. Bartlett, and V. Blanz, "3DShape and 2D Surface Textures of Human Faces: The Role of ,Averages ${ }^{\text {ee }}$ in Attractiveness and Age," Image and Vision Computing,vol. 18, pp. 9-19, 1999.

[5] T. Serre, L.Wolf, and T. Poggio. Object recognition with features inspired by visual cortex. In Proc. IEEE CVPR, pages $994-1000,2005$.

[6] Y. Kwon and N. Lobo, "Age Classification from Facial Images," Proc. IEEE Conf. Computer Vision and Pattern Recognition, pp. 762-767, 1994.

[7] A. Lanitis, C. Draganova, and C. Christodoulou, "Comparing Different Classifiers for Automatic Age Estimation," IEEE Trans. Systems, Man, and Cybernetics Part B, vol. 34, no. 1, pp. 621-628, Feb. 2004.

[8] H. Fukai, H. Takimoto, Y. Mitsukura, and M. Fukumi, "Apparent Age Estimation System Based on Age Perception," Proc. SICE Ann.Conf., pp. 2808-2812, 2007.

[9] A.K. Jain, S.C. Dass, and K. Nandakumar, "Soft Biometric Traits for Personal Recognition Systems," 


\section{International Journal of Science and Research (IJSR) \\ ISSN (Online): 2319-7064}

Index Copernicus Value (2013): 6.14 | Impact Factor (2014): 5.611

Proc. Int 1 Conf. Biometric Authentication, pp. 731-738, 2004.

[10] S. Yan, X. Zhou, M. Liu, M. Hasegawa-Johnson, and T.S. Huang, "Regression from Patch-Kernel," Proc. IEEE Conf. Computer Vision and Pattern Recognition, 2008.

[11]S. Yan, M. Liu, and T.S. Huang, "Extracting Age Information from Local Spatially Flexible Patches," Proc. IEEE Conf. Acoustics, Speech, and Signal Processing, pp. 737-740, 2008.

[12] Electronic Customer Relationship Management (ECRM), http:// en.wikipedia.org/wiki/ECRM, 2010. 